\title{
Knowledge-Aware Neural Networks for Medical Forum Question Classification
}

\author{
Soumyadeep Roy ${ }^{*}$ \\ IIT Kharagpur, India \\ Gunjan Balde \\ IIT Kharagpur, India \\ Megha Khosla \\ L3S Research Center, Germany
}

\author{
Sudip Chakraborty \\ IIT Kharagpur, India \\ Prakhar Sharma \\ IIT Kharagpur, India \\ Shamik Sural \\ IIT Kharagpur, India
}

\author{
Aishik Mandal \\ IIT Kharagpur, India \\ Anandhavelu Natarajan \\ Adobe Research, India \\ Niloy Ganguly* \\ IIT Kharagpur, India
}

\begin{abstract}
Online medical forums have become a predominant platform for answering health-related information needs of consumers. However, with a significant rise in the number of queries and the limited availability of experts, it is necessary to automatically classify medical queries based on a consumer's intention, so that these questions may be directed to the right set of medical experts. Here, we develop a novel medical knowledge-aware BERT-based model (MEDBERT) that explicitly gives more weightage to medical conceptbearing words, and utilize domain-specific side information obtained from a popular medical knowledge base. We also contribute a multi-label dataset for the Medical Forum Question Classification (MFQC) task. MEDBERT achieves state-of-the-art performance on two benchmark datasets and performs very well in low resource settings.
\end{abstract}

\section{CCS CONCEPTS}

- Applied computing $\rightarrow$ Consumer health; $\bullet$ Computing methodologies $\rightarrow$ Supervised learning by classification.

\section{KEYWORDS}

Clinical text classification, online health communities

\section{ACM Reference Format:}

Soumyadeep Roy, Sudip Chakraborty, Aishik Mandal, Gunjan Balde, Prakhar Sharma, Anandhavelu Natarajan, Megha Khosla, Shamik Sural, and Niloy Ganguly. 2021. Knowledge-Aware Neural Networks for Medical Forum Question Classification. In Proceedings of the 30th ACM International Conference on Information and Knowledge Management (CIKM '21), November 15, 2021, Virtual Event, QLD, Australia. ACM, New York, NY, USA, 5 pages. https://doi.org/10.1145/3459637.3482128

*Also affiliated to L3S Research Center, Germany

CIKM '21, November 1-5, 2021, Virtual Event, QLD, Australia

(C) 2021 Copyright held by the owner/author(s). Publication rights licensed to ACM.

This is the author's version of the work. It is posted here for your personal use. Not for redistribution. The definitive Version of Record was published in Proceedings of the 30th ACM International Conference on Information and Knowledge Management (CIKM '21), November 1-5, 2021, Virtual Event, QLD, Australia, https://doi.org/10.1145/3459637.3482128.

\section{INTRODUCTION}

Online medical or health forums have become quite popular and presently act as a reliable source of health-related information, advice or support [25]. Given the limited availability of medical professionals, researchers have developed automated systems to detect the quality of an online health article [4] or to identify the health information need category of a user based on its medical forum question in order to direct it to the relevant medical expert $[7,11]$. In this paper, we focus on the Medical Forum Question Classification (MFQC) task, where the aim is to classify a health forum question (either a query or query plus description) based on the intended health information need category of a user posting it. For example, the target class Treatment is concerned with a specific medical procedure like surgery, while Family Support class corresponds to issues related to a caregiver (instead of a patient) like how to support one's spouse or an ill child $[11,28]$. Currently, MFQC is formulated as a single-label multi-class prediction problem [11]. Existing works for medical question classification usually employ hand-crafted features or pretrained embeddings $[9,17,21,24,28]$ as input representations which result in the loss of context information present in the raw input. Moreover, as these representations are constructed using limited and noisy data (because of large presence of contractions and misspellings as well as vocabulary mismatch with medical professionals), these do not generalize well for the test data.

To overcome the above problems, first, we utilize pretrained models to extract statistically powerful context preserving representations from the raw input data. Pretrained models like BERT [8], GPT-2 [23] and RoBERTa [18] are usually trained on a large number of documents in a self-supervised manner to obtain generalpurpose language representations. Task specific fine-tuning [10] is usually preferred over the feature extraction of pretrained embeddings [22] and has shown vast improvements in several natural language processing tasks [34]. Second, we provide medical domain knowledge as side information to our model, by providing explicit importance to medical concept-bearing words or what we call aspects. This in turn helps us to learn both contextualized as well as a medical domain knowledge-aware representation of a medical forum question. The first standalone, medical knowledgeaware neural model (SoA-DN) to use additional medical domain features (biomedical entities extracted using Metamap [5]) for the MFQC task, fails to achieve high classification performance [11]. In general, several works [13, 20, 29, 30] investigated novel strategies 
to incorporate domain-specific side information present as structured data in knowledge bases or knowledge graphs into neural models (including transformer-based pretrained models).

We make the following contributions in this paper. First, we propose a novel application of dual encoder model [6, 31, 33] for MFQC task. Our approach, MEDBERT, employs BERT encoders to extract global context as well as medical context aware representations of the documents. We additionally enhance our representations by incorporating additional domain-specific side information. In particular, we attribute explicit importance to medical concept bearing terms in our final representations by a simple masking procedure. MEDBERT outperforms the state-of-the-art models across on two benchmark datasets of ICHI and CADEC and particularly works well in low-resource setting (using 30\% training data). Prior studies [11] observe that the target classes of ICHI are mutually overlapping and thus multi-label classification will be a better formulation for MFQC task. However, to the best of our knowledge, there does not exist any multi-label medical forum text classification dataset, and thus rigorously annotate the CADEC dataset [14], a benchmark adverse drug event medical forum dataset across four health information search categories (see Section 3.1); this forms our second contribution. Finally, we perform extensive analysis to better understand the workings of MEDBERT, and make the codebase publicly available at https://github.com/roysoumya/knowledge

\section{METHODOLOGY}

MEDBERT is comprised of four modules (see Figure 1 for methodology overview) - (i) medical term extraction using a medical knowledge base, which serve as domain-specific side information to our model (Section 2.1), (ii) a BERT-based encoder (Bert ${ }_{\text {global }}$ ) that considers both medical words as well as their context (Section 2.2), (iii) a BERT-based encoder (Bert local $_{1}$ ) that explicitly gives more importance to medical concept-bearing words (Section 2.3), and (iv) merging the representations learnt by Bert global $_{\text {and Bert }}$ local (Section 2.4) for final class prediction.
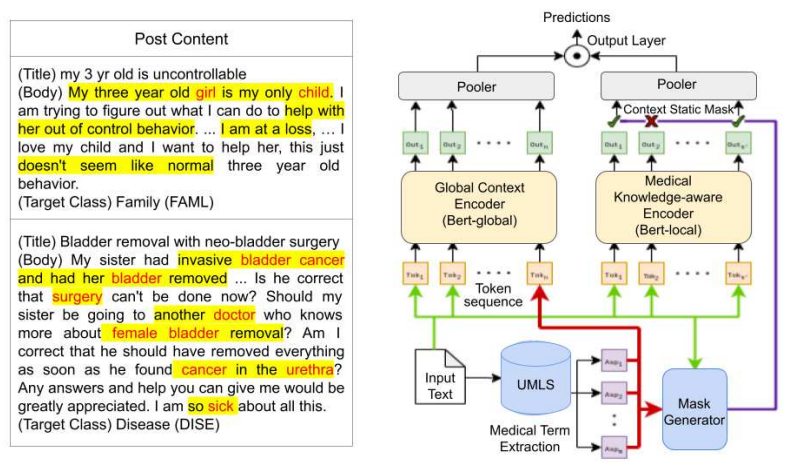

Figure 1: (left) Anecdotal example from ICHI dataset where medical concept-bearing aspects (marked red) are highlighted along with context (right) Methodology overview of MEDBERT for MFQC task

\subsection{Extraction of Medical Aspects}

Here, we describe the identification of medical concept-bearing words in particular, and medically relevant words in general, from the input text. To put it more formally, as previously described, we represent the input text as a sequence of words, $x^{(i)}=w_{1}, w_{2}, \ldots, w_{n}$, we aim to obtain a subsequence from $x^{(i)}, x^{(i, t)}=w_{1}^{t}, w_{2}^{t}, \ldots, w_{m}^{t}$, where $1 \leq m \leq n$. Here, $x^{(i, t)}$ represents the tokens that is (or a part of) a medical concept-bearing word (or phrase).

The aspects are UMLS medical concepts, which are extracted using the QuickUMLS [26] tool. However, we observe that not all extracted medical entities are meaningful for the task at hand. We, thus select only sixteen UMLS Semantic Classes, as used by Jalan et al. [11], that are closely associated with the target classes of MFQC task. As the posts are made by consumers not belonging from medical background, the medical vocabulary used is quite different from that contained in medical knowledge bases like UMLS. Thus we also utilize a medical lexicon known as Patient-Friendly Term List [3], that contains around 1400 MedDRA Lowest Level Terms which include the most frequently used vocabulary by patients or consumers for reporting adverse events. Some examples include Aching in limb, Blood pressure inadequately controlled, Crawling sensation of skin. In the following sections we describe how we incorporate the importance of these terms to learn medical domain specific contextual representations.
sention.

\subsection{Global Context Representation (Bert global )}

To learn a global representation we follow the design of LCF [33]. In particular we append the aspect terms to the original document sequence to serve as input to our global BERT encoder.

Input. The global context sequence for a given medical forum question is constructed in following manner: input text + aspect $_{1}+$ aspect $_{2}+\ldots+$ aspect $_{n}$ where the input text and aspects are separated by the special token [SEP]. The input to BERT encoder will be: $[C L S]+$ 'I had surgery for retinal detachment in December.' + [SEP] + 'surgery' + 'retinal detachment' + [SEP] .

Text encoder (BERT) details. For the MEDBERT model, we use

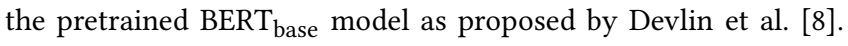
BERT is a multi-layer bidirectional Transformer encoder based on its original implementation as described by Vaswani et al. [27]. Here, we use the uncased variant which ignores the differences in case of a word (like cricket and Cricket). We apply the standard fine-tuning technique [8] where the final hidden vector corresponding to the $[C L S]$ token is used as the aggregate representation for the baseline BERT model.

\subsection{Medical Knowledge-Aware Representation (Bert local $_{\text {lol }}$}

We now describe the steps involved in obtaining the medical domain knowledge-aware representation using BERT encoder, where (i) a deep representation of the input text is generated using the BERT encoder, and then (ii) the deep document representation is modified using a context static mask that forces BERT to prioritize medically-relevant words over the remaining words, thus making it a medical domain knowledge-aware representation. 
Input. The input to the local context sequence consists of simply the sequence of tokens for a given document. We follow the standard method where the tokens are surrounded by [CLS] and [SEP] on the left and right, respectively. For example, if the input text is 'I had surgery for retinal detachment in December.', the input to our encoder will be: $[C L S]+$ I had surgery for retinal detachment in December. $+[S E P]$.

Learning aspect-aware representations Medical features are crucial for improving the performance of medical text-classification and using just the text in isolation fails to capture the required domain knowledge. We treat these features as aspects (marked red in the document shown in Figure 1) which our model should give more attention. To learn aspect aware representations, we adopt a simple feature masking strategy as in [33]. In particular, we apply a context static mask over the output representation of Bert $_{\text {local }}$ encoder such that the output representations corresponding to all words which are not aspects are masked or set to 0 . These retained representations are both domain-specific and contextualized. In essence, the output tokens from the local context encoder are being filtered using masked entity representations. In the above example, for the sentence 'I had surgery for retinal detachment in December', the medical aspects extracted are surgery and retinal detachment. Assuming, each token belongs within the vocabulary, the corresponding context static mask will be a binary vector: [00101100]

\subsection{Class Prediction}

To obtain the final representations, we concatenate the local and global context representation vectors as: $\bar{o}=\left[B E R T_{\text {local }} ; B E R T_{\text {global }}\right]$ It is then passed through a fully-connected layer to project the concatenated vector into the space of the targeted $L$ classes : $\bar{o}_{\text {dense }}=$ $\bar{W}^{T} \bar{o}+\bar{b}$. Finally the posterior class is obtained by applying softmax on $\bar{o}_{\text {dense. }}$. We enforce weight sharing for both the local and global context encoders in MedBERT. MedBERT uses the cross-entropy loss function for single-label tasks and 'binary cross-entropy' for multi-label classification tasks.

\section{EXPERIMENTAL EVALUATION}

We evaluate our proposed MEDBERT model on benchmark dataset as well as create an annotated corpus of the multi-label dataset and also test on it. We run each model for five times and report the mean performance scores in Table 1. We perform the McNemar's test [19] for significance testing.

\subsection{Experimental Setup}

Datasets. We evaluate on two document-level datasets (i) ICHI [1]: It is a single-label multi-class MFQC dataset, comprising of questions posted on health discussion forums. It consists of 8000 training and 3000 test data points. Each data point consists of a question title, question text and label (belonging to one of seven health information need categories like Demographic (DEMO), Disease (DISE), Treatment (TRMT), Goal-oriented (GOAL), Pregnancy (PREG), Family Support (FMLY), Socializing (SOCL)). The dataset is balanced and we use the standard train-test splits employed by prior studies $[11,28]$. (ii) CADEC: It is the first multi-label MFQC dataset, comprising of 942 training and 300 testing data points. We utilize a benchmark annotated corpus [14] consisting of pharmacovigilance-related consumer questions/reviews, and use two computer-science graduate students who are very familiar with online forums but limited exposure to online medical forums, to label the data points into four health information search categories consisting of Uncertainty of post-diagnosis (UPD), Medical Assistance (MAS), Diet and maintenance (DM), and Information source (IS) (appropriate for posts related to 'adverse drug events'), following the same guidelines as Derksen et al. [7]; we remove data points where two annotators disagree with each other. The dataset suffers from high class imbalance and thus during training, multi-class oversampling is used where minority classes are selected one-by-one and then oversampled to the cardinality of the initial majority class.

Baselines. We compare with three types of baseline models - (i) non-neural models like TFIDF + SVM, (ii) standard neural models based on pretrained embeddings that do not use medical domain knowledge like FastText [12], TextCNN [15], HAN [32] and ICHI 2016 Challenge Winners [28] (iii) medical domain knowledgeaware neural models like SoA-DN and H-BiLSTM + TFIDF-DN + SoA-DN, model variants developed by Jalan et al. [11]; we are unable to reproduce their results and thus report their overall accuracy scores, the only metric reported in their paper, (iv) medical domain knowledge-aware transformer-based pretrained models like Local Context Focus Bert (LCF-BERT) [33], Bert-base [8], MEDBERT variants (Global only, Local only required for ablation study purposes).

Training Details. Here, we use the BERT implementation of transformers [2] library. For preprocessing BERT-based models, we perform lowercasing and set maximum sequence length to 256 tokens with longer documents truncated from the right. Whereas for nonBERT based models, each word is converted to lower case and punctuations are removed, but stopwords are retained; the words are also lemmatized, and maximum document length is set to 3000 words. We perform hyperparameter-tuning for MedBERT in terms of learning rate ( $5 e-5,3 e-5,2 e-5)$, batch size (16 and 32) and number of epochs for fine-tuning ( 2 to 10 epochs). MEDBERT model used in our experiments is trained with a batch size of 16, Adam as optimizer with a starting learning rate of $5 \mathrm{e}-5$, linearly decayed throughout training and dropout rate of 0.1 . The fine-tuning is performed for 2 epochs in case of ICHI and 10 epochs for CADEC. For the baseline models, we use the default hyperparameter values.

\subsection{Performance Evaluation}

We evaluate the classification models in terms of accuracy and F1score (preferred more in case of class-imbalanced data like CADEC). Discussion of Results. Our proposed model, MEDBERT achieves the highest performance in terms of both ICHI and CADEC dataset, which is shown in Table 1. We observe that the Bert-base model is able to outperform the state-of-the-art model [11] for the ICHI data, without using any medical features. We perform comparably with the 3-component ensemble model of Jalan et al. [11] comprising of TF-IDF, medical domain features like SoA-DN (second row of Table 1), and a neural network model (Hierarchical BiLSTM or HAN). However, we outperform their standalone 'SoA-DN' model 
Table 1: Performance comparison of MEDBERT and baseline models. * indicates second best-performing model.

\begin{tabular}{|c|c|c|c|c|c|c|c|c|c|c|c|}
\hline \multirow[b]{3}{*}{ Models } & \multirow{3}{*}{$\begin{array}{l}\text { ICHI } \\
\text { ALL } \\
\text { Acc }\end{array}$} & \multicolumn{10}{|c|}{ CADEC } \\
\hline & & \multicolumn{2}{|c|}{ UPD } & \multicolumn{2}{|c|}{ MAS } & \multicolumn{2}{|c|}{$\mathrm{DM}$} & \multicolumn{2}{|c|}{ IS } & \multicolumn{2}{|c|}{ ALL } \\
\hline & & Acc & F1 & Acc & F1 & Acc & F1 & Acc & F1 & Acc & F1 \\
\hline ICHI 2016 & 0.68 & - & - & - & - & - & - & - & - & - & - \\
\hline Challenge & & & & & & & & & & & \\
\hline Winners [28] & & & & & & & & & & & \\
\hline SoA-DN [11] & 0.6 & - & - & - & - & - & - & - & - & - & - \\
\hline H-BLSTM + & 0.7 & - & - & - & - & - & - & - & - & - & - \\
\hline TFIDF-DN + & & & & & & & & & & & \\
\hline SoA-DN [11] & & & & & & & & & & & \\
\hline TFIDF + SVM & 0.64 & 0.82 & $0.59^{*}$ & 0.83 & 0.7 & 0.91 & 0.74 & 0.92 & 0.58 & 0.87 & 0.65 \\
\hline FastText & 0.61 & 0.86 & 0.46 & 0.81 & 0.45 & 0.88 & 0.47 & 0.82 & 0.48 & 0.84 & 0.47 \\
\hline TextCNN & 0.58 & 0.83 & 0.47 & 0.87 & 0.72 & 0.9 & 0.69 & 0.92 & 0.52 & 0.88 & 0.6 \\
\hline HAN & 0.61 & 0.83 & 0.6 & 0.82 & 0.6 & 0.88 & 0.61 & 0.92 & 0.48 & 0.86 & 0.57 \\
\hline LCF-BERT & $0.69^{*}$ & 0.86 & 0.46 & 0.81 & 0.45 & 0.88 & 0.47 & 0.92 & 0.48 & 0.87 & 0.47 \\
\hline MEDBERT & 0.7 & 0.84 & 0.55 & 0.88 & 0.74 & 0.92 & $0.8^{*}$ & 0.95 & 0.75 & 0.9 & 0.71 \\
\hline Bert-base & 0.65 & 0.84 & 0.32 & 0.86 & 0.72 & 0.91 & 0.52 & 0.94 & 0.54 & 0.89 & 0.53 \\
\hline $\begin{array}{l}\text { MEDBERT } \\
\text { (Global only) }\end{array}$ & 0.68 & 0.79 & 0.55 & 0.86 & 0.74 & 0.93 & 0.81 & 0.94 & 0.73 & 0.88 & 0.71 \\
\hline $\begin{array}{l}\text { MEDBERT } \\
\text { (Local only) }\end{array}$ & 0.68 & 0.78 & 0.55 & 0.87 & 0.72 & 0.91 & 0.77 & 0.94 & $0.74^{*}$ & 0.88 & 0.7 \\
\hline
\end{tabular}

by $16.7 \%$. The performance difference between MEDBERT and variants versus the competing baseline models is statistically significant in terms of McNemar's test, for all models except LCF-BERT for ICHI data. However, on CADEC dataset, we observe that the MEDBERT outperforms LCF-BERT by a significant margin (average F1 score of $0.71 \mathrm{v} / \mathrm{s} 0.47$ ). We also notice that the classification accuracy of all models improved dramatically (average model accuracy increased from 0.65 to 0.84 ) on the multi-label dataset of CADEC as compared to ICHI data (11.3\% of training data have more than one label as positive), implying that MFQC is better suited as a multi-label classification task.

Ablation Analysis. For ICHI data, MEDBERT significantly outperforms MEDBERT (Global only) and MEDBERT (Local only) models by $2.9 \%$, thus highlighting the importance of the dual encoder architecture in our case. However, in case of CADEC, MEDBERT along with Global only and Local only variants, perform comparably. This may be attributed to the fact that the medical concepts filtered by the medical aspect extraction module of MEDBERT, do not completely accommodate CADEC information need categories that are focused on a sub-domain of adverse drug events only. Next, we observe that MEDBERT outperforms Bert-base (may be thought of as MEDBERT w/o medical keywords) by $7.7 \%$, and shows the importance of adding medical side-information to these pretrained models.

Error Analysis on ICHI. From Figure 2, we observe that MEDBERT fails most frequently in the following cases (true $\rightarrow$ predicted): PREG $\rightarrow$ DEMO, DEMO $\rightarrow$ PREG, and SOCL $\rightarrow$ DISE. On manual inspection and even from definition of both classes, we observe that 'PREG' class targets a specific demographic, both age and gender, where topics like difficulties with conception, mother and unborn child's health during pregnancy, are covered. Thus, by definition, all data points labeled as 'PREG', should also hold true for 'DEMO'. Contrary to other target classes, Socializing (SOCL) do not target any health-related issues and rather focuses on recreational activities or hobbies. Thus, forcing MEDBERT to focus on medical concept-bearing words does not contribute towards improving the classifier performance of SOCL class.

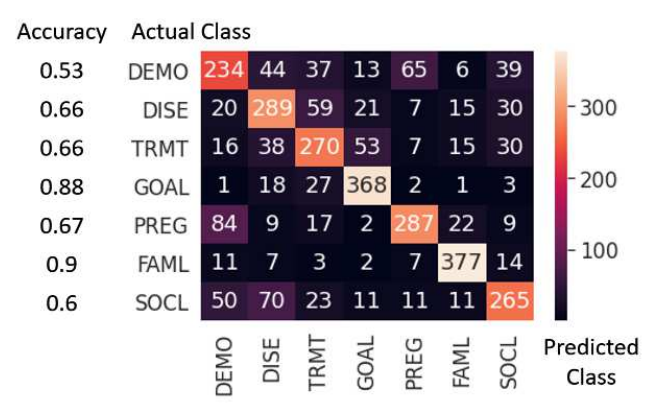

Figure 2: Confusion matrix of MEDBERT on ICHI data along with class-wise accuracy scores, highlighting the most frequent misclassifications: PREG $\rightarrow$ DEMO, DEMO $\rightarrow$ PREG, and SOCL $\rightarrow$ DISE

Effect of Training Data Size. To evaluate the generalization power of different models, we evaluate them on different training data sizes of ICHI dataset (see Figure 3). First, we observe that LCFBERT and MEDBERT outperform other methods by a large margin. For small train-sets, these approaches leverage the knowledge from pretrained models and use the training data only for finetuning the representations. Further MEDBERT outperforms LCFBert which showcase the advantage of using domain specific additional side information in the form of medical terms or aspects. Specifically for $30 \%$ training data, MEDBERT outperforms LCF-BERT by $4.76 \%$.

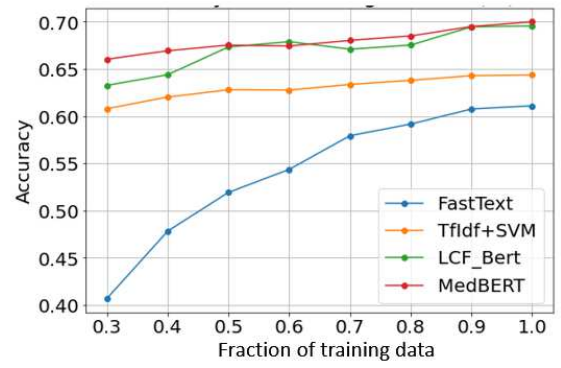

Figure 3: Performance comparison of MEDBERT and baselines for different fractions of training data on ICHI data

\section{CONCLUSION}

We propose MEDBERT, a novel application of transformers-based dual encoder model, for MFQC task, which is also medical domain knowledge-aware. We contribute a multi-label MFQC dataset; MEDBERT achieves state-of-the-art performance on ICHI (accuracy of 0.7 ) and CADEC dataset (accuracy of 0.9 and macro F1 score of 0.71 ), and generalizes very well in low-resource settings. Through extensive experimentation, we learn that incorporating medical concept-bearing terms as side information, contribute significantly to MEDBERT. We learn that certain target classes heavily depend on keywords, while others require one to learn optimal representation of medical context. An interesting future direction will 
be to extend MEDBERT to structured prediction tasks like entity and relation prediction, or broadly link prediction. Instead of BERT, we will work with BioBERT [16], which is a domain-specific pretrained model trained on biomedical articles.

Acknowledgements. This work is supported in part by the Institute PhD Fellowship of IIT Kharagpur, the Federal Ministry of Education and Research (BMBF), Germany under the project LeibnizKILabor (grant no. 01DD20003), the Adobe-funded project titled "Computational Aspects and Role of Content for Persuasive Brand Positioning”, and IMPRINT-1 Project RCO (project no. 6537).

\section{REFERENCES}

[1] 2016. Healthcare data analytics challenge. In 2016 IEEE International Conference on Healthcare Informatics (ICHI). xli-xli. https://doi.org/10.1109/ICHI.2016.111

[2] 2020. Huggingface Transformers. https://huggingface.co/transformers/

[3] 2020. MedDRA Patient-Friendly Term List (Version: 23.0). https://www.meddra.org/patient-friendly-term-list

[4] Fariha Afsana, Muhammad Ashad Kabir, Naeemul Hassan, and Manoranjan Paul. 2021. Automatically Assessing Quality of Online Health Articles. IEEE Journal of Biomedical and Health Informatics 25, 2 (2021), 591-601. https://doi.org/10.1109/JBHI.2020.3032479

[5] Alan R Aronson. 2001. Effective mapping of biomedical text to the UMLS Metathesaurus: the MetaMap program.. In Proceedings of the AMIA Symposium. 17. https://pubmed.ncbi.nlm.nih.gov/11825149

[6] Rajarshi Bhowmik, Karl Stratos, and Gerard de Melo. 2021. Fast and Effective Biomedical Entity Linking Using a Dual Encoder. In Proceedings of the 12th International Workshop on Health Text Mining and Information Analysis. online, 28-37. https://www.aclweb.org/anthology/2021.louhi-1.4

[7] Christina Derksen, Anna Serlachius, Keith J. Petrie, and Nicola Dalbeth. 2017 "What say ye gout experts?" a content analysis of questions about gout posted on the social news website Reddit. BMC Musculoskelet Disord 18, 1 (23 Nov 2017), 488-488. https://doi.org/10.1186/s12891-017-1856-y

[8] Jacob Devlin, Ming-Wei Chang, Kenton Lee, and Kristina Toutanova. 2019. BERT: Pre-training of Deep Bidirectional Transformers for Language Understanding. In Proceedings of the 2019 Conference of the North American Chapter of the Association for Computational Linguistics: Human Language Technologies, Volume 1 (Long and Short Papers). Minneapolis, Minnesota, 4171-4186. https://doi.org/10.18653/v1/N19-1423

[9] Haihong Guo, Xu Na, Li Hou, and Jiao Li. 2017. Classifying Chinese Questions Related to Health Care Posted by Consumers Via the Internet. 7 Med Internet Res 19, 6 (20 Jun 2017), e220. https://doi.org/10.2196/jmir.7156

[10] Jeremy Howard and Sebastian Ruder. 2018. Universal Language Model Finetuning for Text Classification. In Proceedings of the 56th Annual Meeting of the Association for Computational Linguistics (Volume 1: Long Papers). 328-339. https://doi.org/10.18653/v1/P18-1031

[11] Raksha Jalan, Manish Gupta, and Vasudeva Varma. 2018. Medical forum question classification using deep learning. In European Conference on Information Retrieval. Springer, 45-58. https://doi.org/10.1007/978-3-319-76941-7_4

[12] Armand Joulin, Edouard Grave, Piotr Bojanowski, and Tomas Mikolov. 2017. Bag of Tricks for Efficient Text Classification. In Proceedings of the 15th Conference of the European Chapter of the Association for Computational Linguistics: Volume 2, Short Papers. Valencia, Spain, 427-431. https://aclanthology.org/E17-2068

[13] Annervaz K M, Somnath Basu Roy Chowdhury, and Ambedkar Dukkipati. 2018 Learning beyond Datasets: Knowledge Graph Augmented Neural Networks for Natural Language Processing. In Proceedings of the 2018 Conference of the North American Chapter of the Association for Computational Linguistics: Human Language Technologies, Volume 1 (Long Papers). New Orleans, Louisiana, 313-322. https://doi.org/10.18653/v1/N18-1029

[14] Sarvnaz Karimi, Alejandro Metke-Jimenez, Madonna Kemp, and Chen Wang. 2015. Cadec: A corpus of adverse drug event annotations. Fournal of Biomedical Informatics 55 (2015), 73 - 81. https://doi.org/10.1016/j.jbi.2015.03.010

[15] Yoon Kim. 2014. Convolutional Neural Networks for Sentence Classification In Proceedings of the 2014 Conference on Empirical Methods in Natural Language Processing. 1746-1751. https://doi.org/10.3115/v1/d14-1181

[16] Jinhyuk Lee, Wonjin Yoon, Sungdong Kim, Donghyeon Kim, Sunkyu Kim, Chan Ho So, and Jaewoo Kang. 2019. BioBERT: a pre-trained biomedical language representation model for biomedical text mining. Bioinformatics 36, 4 (09
2019), 1234-1240. https://doi.org/10.1093/bioinformatics/btz682

[17] Feifan Liu, Lamont D. Antieau, and Hong Yu. 2011. Toward automated consumer question answering: Automatically separating consumer questions from professional questions in the healthcare domain. Fournal of Biomedical Informatics 44, 6 (2011), 1032 - 1038. https://doi.org/10.1016/j.jbi.2011.08.008

[18] Yinhan Liu, Myle Ott, Naman Goyal, Jingfei Du, Mandar Joshi, Danqi Chen, Omer Levy, Mike Lewis, Luke Zettlemoyer, and Veselin Stoyanov. 2019. Roberta: A robustly optimized bert pretraining approach. arXiv preprint arXiv:1907.11692 (2019).

[19] Quinn McNemar. 1947. Note on the sampling error of the difference between correlated proportions or percentages. Psychometrika 12, 2 (1947), 153-157. https://doi.org/10.1007/BF02295996

[20] Todor Mihaylov and Anette Frank. 2018. Knowledgeable Reader: Enhancing Cloze-Style Reading Comprehension with External Commonsense Knowledge. In Proceedings of the 56th Annual Meeting of the Association for Computational Linguistics (Volume 1: Long Papers). Melbourne, Australia, 821-832. https://doi.org/10.18653/v1/P18-1076

[21] Yassine Mrabet, Halil Kilicoglu, Kirk Roberts, and Dina Demner-Fushman. 2016. Combining open-domain and biomedical knowledge for topic recognition in consumer health questions. In AMIA Annual Symposium Proceedings, Vol. 2016. 914. https://pubmed.ncbi.nlm.nih.gov/28269888/

[22] Matthew Peters, Mark Neumann, Mohit Iyyer, Matt Gardner, Christopher Clark, Kenton Lee, and Luke Zettlemoyer. 2018. Deep Contextualized Word Representations. In Proceedings of the 2018 Conference of the North American Chapter of the Association for Computational Linguistics: Human Language Technologies, Volume 1 (Long Papers). 2227-2237. https://doi.org/10.18653/v1/N18-1202

[23] Alec Radford, Jeffrey Wu, Rewon Child, David Luan, Dario Amodei, and Ilya Sutskever. 2019. Language models are unsupervised multitask learners. (2019). https://www.persagen.com/files/misc/radford2019language.pdf

[24] Kirk Roberts, Laritza Rodriguez, Sonya E Shooshan, and Dina DemnerFushman. 2016. Resource Classification for Medical Questions. AMIA. Annual Symposium proceedings. AMIA Symposium 2016 (2016), 1040-1049. https://europepmc.org/articles/PMC5333297

[25] Ashnish Sinha, Tom Porter, and Andrew Wilson. 2018. The Use of Online Health Forums by Patients With Chronic Cough: Qualitative Study. 7 Med Internet Res 20, 1 (24 Jan 2018), e19. https://doi.org/10.2196/jmir.7975

[26] Luca Soldaini and Nazli Goharian. 2016. Quickumls: a fast, unsupervised approach for medical concept extraction. Medical Information Retrieval (MedIR) Workshop. http://medir2016.imag.fr/data/MEDIR_2016_paper_16.pdf

[27] Ashish Vaswani, Noam Shazeer, Niki Parmar, et al. 2017. Attention is All you Need. In Advances in Neural Information Processing Systems 30. Curran Associates, Inc., 5998-6008. http://papers.nips.cc/paper/7181-attention-is-all-you-need.pdf

[28] Janu Verma, Bum Chul Kwon, Yu Cheng, Soumya Ghosh, and Kenney Ng. 2016. Classification of healthcare forum messages.

[29] Dirk Weissenborn, Tomáš Kočiskỳ, and Chris Dyer. 2017. Dynamic integration of background knowledge in neural NLU systems. arXiv preprint arXiv:1706.02596 (2017)

[30] Bishan Yang and Tom Mitchell. 2017. Leveraging Knowledge Bases in LSTMs for Improving Machine Reading. In Proceedings of the 55th Annual Meeting of the Association for Computational Linguistics (Volume 1: Long Papers). Vancouver, Canada, 1436-1446. https://doi.org/10.18653/v1/P17-1132

[31] Heng Yang, Biqing Zeng, Jianhao Yang, Youwei Song, and Ruyang Xu. 2021. A multi-task learning model for Chinese-oriented aspect polarity classification and aspect term extraction. Neurocomputing 419 (2021), 344-356. https://doi.org/10.1016/j.neucom.2020.08.001

[32] Zichao Yang, Diyi Yang, Chris Dyer, Xiaodong He, Alex Smola, and Eduard Hovy. 2016. Hierarchical Attention Networks for Document Classification. In Proceedings of the 2016 Conference of the North American Chapter of the Association for Computational Linguistics: Human Language Technologies. San Diego, California, 1480-1489. https://doi.org/10.18653/v1/N16-1174

[33] Biqing Zeng, Heng Yang, Ruyang Xu, Wu Zhou, and Xuli Han. 2019. LCF: A Local Context Focus Mechanism for Aspect-Based Sentiment Classification. Applied Sciences 9, 16 (2019). https://doi.org/10.3390/app9163389

[34] Kelly Zhang and Samuel Bowman. 2018. Language Modeling Teaches You More than Translation Does: Lessons Learned Through Auxiliary Syntactic Task Analysis. In Proceedings of the 2018 EMNLP Workshop BlackboxNLP: Analyzing and Interpreting Neural Networks for NLP. 359-361. https://doi.org/10.18653/v1/W18-5448 